\title{
Sea-level rise could overwhelm coral reefs
}

\author{
An assessment of the capacity of coral reefs to grow fast enough to keep up with \\ projected rises in sea level finds that most reefs will fall behind if nothing is done \\ to restore them. SEE ARTICLE P.396
}

\section{ILSA B. KUFFNER}

$\mathrm{C}$ oral reefs are famous for housing biodiversity and attracting tourists, and the economic benefits that reefs provide for tropical, coastal communities around the globe measure in the billions of dollars ${ }^{1}$. One of the main services provided by reefs is that they act as natural breakwaters (Fig. 1), protecting shorelines and human-built infrastructure from storms. On page 396, Perry et al. ${ }^{2}$ report a detailed analysis of the ability of coral reefs in two ocean basins to keep growing upwards in the face of the ecological degradation they have already experienced, and taking into account future sea-level rise. The findings show that, as living coral populations wane, their capacity to build reefs might be diminished to the point at which the reef community fails to keep up with the rising ocean surface.

Corals are simple invertebrates that are related to sea anemones and jellyfish, but a trait that sets them apart is their ability to create rock from sea water. Each year, corals add a new layer of calcium carbonate on top of their existing exoskeletons, growing larger and intertwining over thousands of years to form a coastal barrier capable of quelling enormous amounts of wave energy. Corals are under threat from warming oceans and from an onslaught of localized environmental pressures, which collectively cause coral bleaching, slower growth, disease and death ${ }^{3}$. If there are not enough corals alive to keep a reef growing, then erosion takes over and the reef loses elevation.

There are some uncertainties associated with understanding the fate of coral reefs as geological structures. Coral growth does not translate millimetre for millimetre into vertical reef growth. Many constructional and erosional processes are at work simultaneously, adding to and subtracting from the net amount of calcium carbonate produced or lost (the carbonate budget), and determining whether a reef builds or winnows away. In previously published work, Perry and colleagues ${ }^{4}$ were among the first to use field data that account for the organisms responsible for reef building (corals and calcifying algae) and reef breakdown (excavating parrotfish, sea urchins and reef-infesting sponges) in budgeting projections of reef growth and destruction.

In the current work, Perry et al. take those budgeting efforts a step further by combining them with projections of sea-level rise under

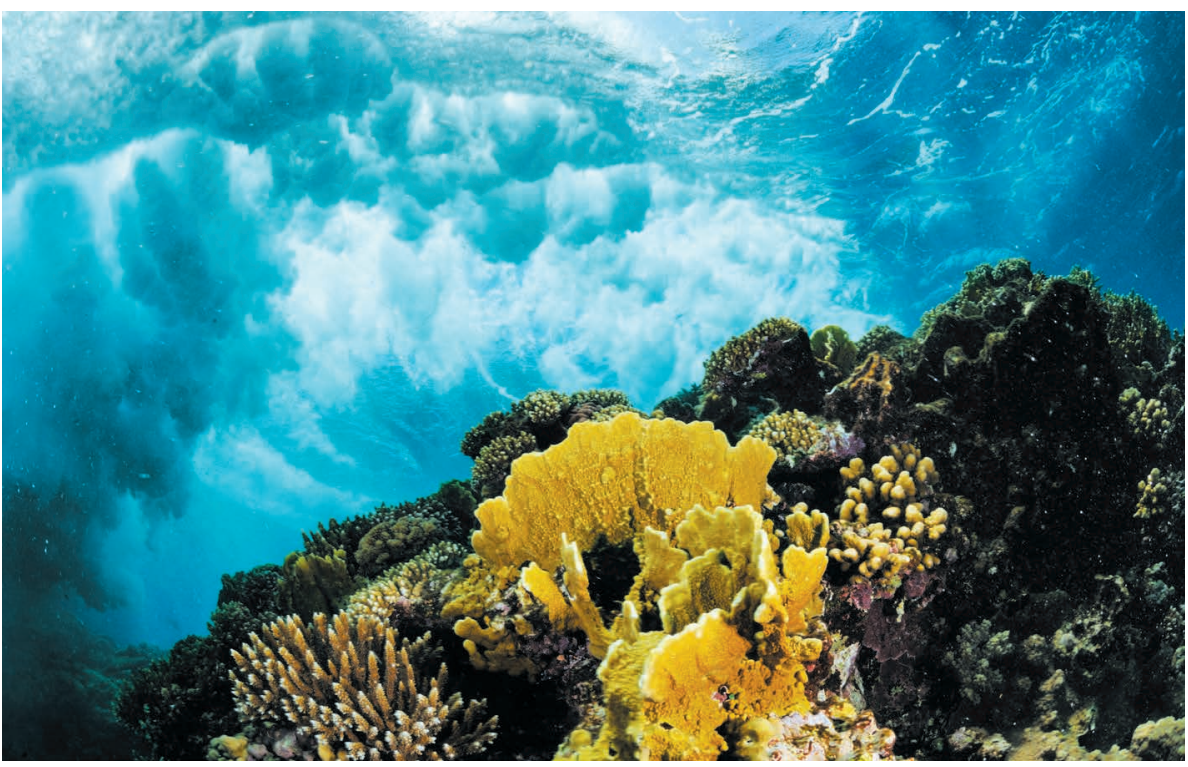

Figure 1 | Natural breakwaters. Coral reefs, such as this one in the Red Sea off the coast of Saudi Arabia, protect shorelines from stormy seas. Perry et al. ${ }^{2}$ report that most coral reefs might not be able to grow fast enough to keep up with future sea-level rises, putting crucial coastal defences at risk. two scenarios published in the Fifth Assessment Report $^{5}$ from the Intergovernmental Panel on Climate Change (IPCC). What they find is not encouraging: 16 reef areas in the tropical western Atlantic Ocean and 6 in the Indian Ocean are barely keeping up with the present sea level. Even worse, only $9 \%$ of the 202 reefs they assessed have the capacity to keep up with the rates of sea-level rise associated with even the more optimistic of the two scenarios (IPCC Representative Concentration Pathway 4.5), which predicts that atmospheric greenhousegas emissions will peak around 2040.

The authors acknowledge that their accounting does not adequately address some reef processes that are important in the budget. If you used a rotary drill to take a peek inside a reef, you would find that only about half of the structure is composed of intact coral skeletons; the rest is either void space or reef detritus, including rubble and sediment ${ }^{6}$. The processes that control the breakdown of reefs and determine whether the resulting material fills the cracks and crevices or gets swept away are not well studied. Although Perry and colleagues' budget did account for the biologically mediated erosion responsible for producing reef rubble and sediments, they did not factor in the chemical dissolution of carbonates, nor evidence suggesting ${ }^{7,8}$ that both of these processes will be accelerated by increased levels of carbon dioxide absorption by the ocean (ocean acidification).

Additionally, the transport of loose sediment away from reefs and into deeper water is largely driven by sporadic storms. This makes it difficult to estimate an average rate at which sediment contributes to reef building. Moreover, sediment transport away from reefs might increase as cyclones become more intense as a result of ocean warming ${ }^{9}-\mathrm{a}$ factor that was also not considered by the authors. Taken together, the processes not accounted for by Perry and colleagues could mean that the projections of reef-building rates are, if anything, too optimistic.

The implications of the study are dire: many island nations and territories are set to quickly lose crucial natural resources responsible for coastal defence. Prompt action is warranted to slow and reverse this loss. Fortunately, reef restoration has come a long way since the twentieth century, when piles of discarded car tyres and engine blocks were used as artificial reefs. Restoration using live corals farmed in offshore nurseries is fast becoming more common and feasible as coral-gardening techniques have been streamlined. So far, these efforts have been driven largely by conservation organizations and hoteliers, but reef-restoration programmes are poised to benefit from initiatives that coordinate restoration practitioners, scientists, governments, resource managers and local communities (see, for example, go.nature. com/2rljaqh).

The feasibility and efficacy of enhancing coastal and community resilience through 
live-coral planting have not been quantified, but Perry et al. provide convincing evidence that the time has come to make reef restoration a priority. A recent analysis ${ }^{10}$ indicates that ecological restoration projects aimed at protecting shorelines might be more costeffective than conventional projects that use engineered concrete structures. Although it is uncertain how much time we can buy for coral reefs through restoration, such projects might extend the existence of the reefs long enough to bridge the gap until global efforts start to decrease the concentration of atmospheric greenhouse gases, thereby slowing the rates of global warming and sea-level rise.

Ilsa B. Kuffner is at the US Geological Survey, St. Petersburg Coastal and Marine Science Center, St. Petersburg, Florida 33701, USA. e-mail:ikuffner@usgs.gov

1. Costanza, R. et al. Nature 387, 253-260 (1997).

2. Perry, C. T. et al. Nature 558, 396-400 (2018).

3. Hughes, T. P. et al. Nature 546, 82-90 (2017).

4. Perry, C. T. et al. Coral Reefs $\mathbf{3 1}, 853-868$ (2012).

5. Church, J. A. et al. in Climate Change 2013: The Physical Science Basis. Contribution of Working
Group I to the Fifth Assessment Report of the Intergovernmental Panel on Climate Change (eds Stocker, T. F. et al.) Ch. 13 (Cambridge Univ. Press, 2013).

6. Hubbard, D. K., Miller, A. I. \& Scaturo, D. J. Sedimentol. Petrol. 60, 335-360 (1990).

7. Tribollet, A., Godinot, C., Atkinson, A. \& Langdon, C. Global Biogeochem. Cycles 23, GB3008 (2009).

8. Silbiger, N. J. \& Donahue, M. J. Biogeosciences 12 567-578 (2015)

9. Webster, P. J., Holland, G. J., Curry, J. A. \& Change, H.-R. Science 309, 1844-1846 (2005)

10.Morris, R. L., Konlechner, T. M., Ghisalberti, M. \& Swearer, S. E. Glob. Change Biol. 24, 1827-1842 (2018).

This article was published online on 13 June 2018.

\section{Frictionless when flat}

\section{Gas transport in nanoscale channels that have perfectly flat walls has been found to be frictionless, challenging the classical theory of gas flow. The findings might enable new devices for gas separation and flow control. SEE LETTER P.420}

\section{CHUANHUA DUAN}

I classical physics, frictionless gas flow through channels is a phenomenon that can occur only under ideal conditions. A key requirement is that the gas molecules undergo specular reflection from the channel walls they rebound so that their angle of incidence is the same as the angle of reflection. In reality, gas molecules are thought to rebound from walls in all directions, a behaviour known as diffuse reflection. But can diffuse reflection be switched to specular reflection to achieve frictionless gas transport, and, if so, what are the fundamental requirements for this? On page 420 , Keerthi et al. ${ }^{1}$ answer these questions by investigating gas transport through two-dimensional, nanometre-scale channels that have atomically flat walls.

Achieving specular reflection for gas molecules seems an impossible task. Even the best, artificially polished surface is randomly bumpy at the tiny scales associated with gas molecules (which are about $10^{-9}-10^{-10}$ metres in diameter). It is this bumpiness that is thought to make gas molecules rebound in all directions, causing diffuse reflection. The concept of diffuse reflection underpins Knudsen theory, which has generally provided a good description of gas transport through channels. But what happens if the channel surfaces are genuinely flat?

To answer this question, Keerthi et al. prepared nanochannels ${ }^{2,3}$ from materials that consist of stacked, 2D layers of atoms: graphite, hexagonal boron nitride (h-BN) and molybdenum disulfide $\left(\mathrm{MoS}_{2}\right)$. These materials can be cleaved to form $2 \mathrm{D}$ crystals that are as thin as a single layer of atoms, and have atomically flat surfaces. $2 \mathrm{D}$ crystals made from different materials can easily stack together, because strong van der Waals forces form between their flat surfaces ${ }^{4}$. The authors made their nanochannels by sandwiching a $2 \mathrm{D}$ crystal containing a narrow gap between two other 2D crystals (Fig. 1). The channels are well sealed, and their heights can be precisely controlled by the number of layers of atoms in the middle $2 \mathrm{D}$ crystal.
The researchers measured the flow of gas through the nanochannels under low pressure. Under these conditions, the gas molecules collide mostly with the surfaces of the channels, rather than with each other. The authors found that the permeability of helium through nanochannels made from graphite or h-BN, whose surfaces are flat even at scales of 1 ångström $\left(10^{-10} \mathrm{~m}\right)$, is ten to several huntheory. Furthermore, they observed that the highest helium permeability measured in graphite nanochannels was independent of the channel length, indicating that there is no momentum loss in the channels and that frictionless flow has been achieved.

By contrast, the permeability of helium through $\mathrm{MoS}_{2}$ nanochannels was the same as that predicted by Knudsen theory. This is dred times higher than predicted by Knudsen

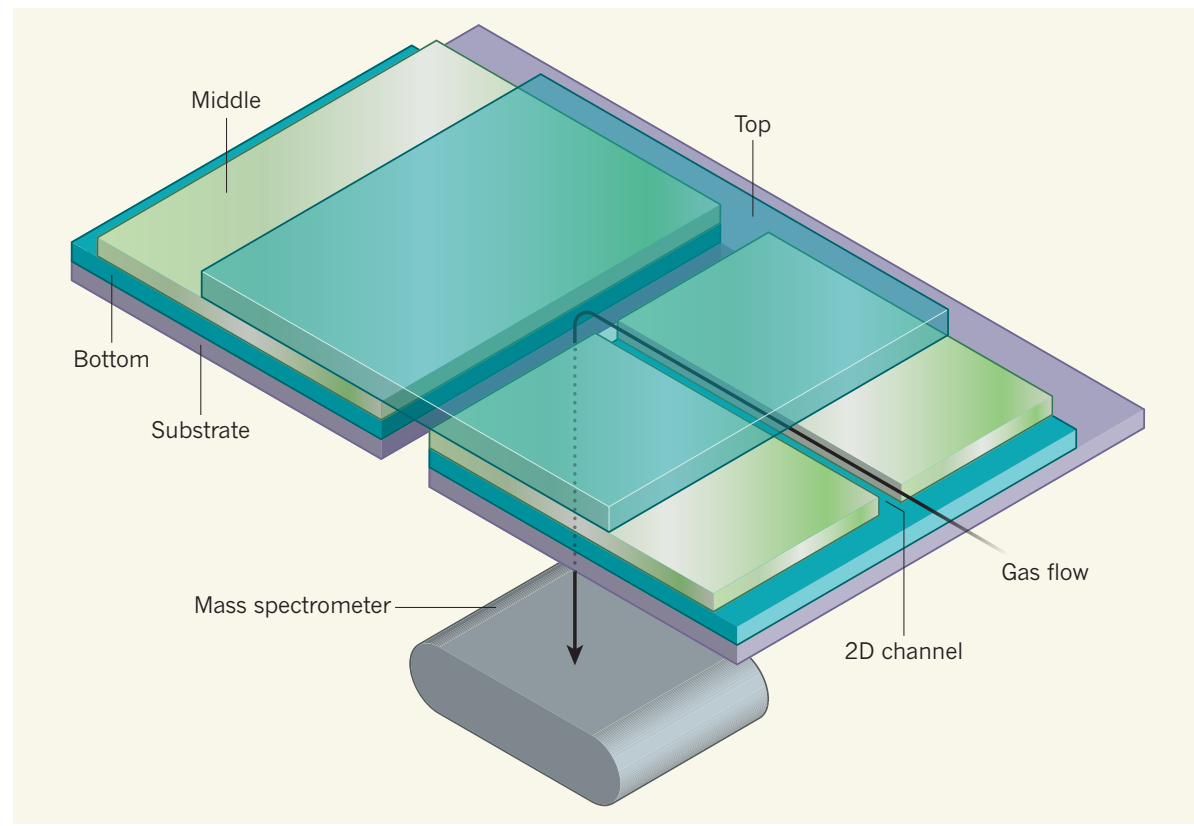

Figure 1 | Measuring gas flow though 2D channels. Keerthi et al. ${ }^{1}$ prepared nanochannels from materials that can be cleaved to produce 2D crystals just one or a few layers of atoms thick. The nanochannels were made by sandwiching a 2D crystal (middle layer) containing a narrow gap between two other $2 \mathrm{D}$ crystals (top and bottom layers). The authors then measured the flow of gas - helium, hydrogen or deuterium - through the channels using a mass spectrometer. In channels made of graphite and hexagonal boron nitride, they observed much higher gas flow than is predicted by classical theory; this flow was confirmed to be frictionless in the graphite channels. However, gas flow conforming to classical theory was observed in channels made of molybdenum disulfide. The properties of the flow seem to depend on the roughness of the channel surfaces as 'perceived' by the gas molecules. 